OPEN ACCESS

Edited by:

Tamer Demiralp,

Istanbul University, Turkey

Reviewed by:

Hakan Gurvit,

Istanbul University, Turkey

Gabriel Gonzalez-Escamilla,

Universitätsmedizin Mainz, Germany

${ }^{*}$ Correspondence:

Kuncheng $L$

cjr.likuncheng@vip.163.com

tThese authors have contributed equally to this work

Received: 25 May 2018 Accepted: 24 September 2018 Published: 29 October 2018

Citation:

Li H, Jia X, Qi Z, Fan X, Ma T, Pang R, Ni H, Li C-sR, Lu J and Li K (2018) Disrupted Functional

Connectivity of Cornu Ammonis

Subregions in Amnestic Mild Cognitive Impairment: A Longitudinal

Resting-State fMRI Study.

Front. Hum. Neurosci. 12:413. doi: 10.3389/fnhum.2018.00413

\section{Disrupted Functional Connectivity of Cornu Ammonis Subregions in Amnestic Mild Cognitive Impairment: A Longitudinal Resting-State fMRI Study}

\author{
Hui Li',2t, Xiuqin Jia ${ }^{3,4 t}$, Zhigang Qi',2, Xiang Fan', Tian Ma', Ran Pang ${ }^{1}$, Hong $\mathrm{Ni}^{1}$, \\ Chiang-shan R. Li5,6,7, Jie Lu ${ }^{1,2}$ and Kuncheng $L i^{1,2 *}$
}

\begin{abstract}
' Department of Radiology, Xuanwu Hospital, Capital Medical University, Beijing, China, ${ }^{2}$ Beijing Key Laboratory of MRI and Brain Informatics, Beijing, China, ${ }^{3}$ Department of Radiology, Beijing Chaoyang Hospital, Capital Medical University, Beijing, China, ${ }^{4}$ Institute of Psychology, Chinese Academy of Sciences, Beijing, China, ${ }^{5}$ Department of Psychiatry, Yale School of Medicine, Yale University, New Haven, CT, United States, ${ }^{6}$ Department of Neuroscience, Yale School of Medicine, Yale University, New Haven, CT, United States, ${ }^{7}$ Beijing Huilongguan Hospital, Beijing, China
\end{abstract}

Background: The cornu ammonis (CA), as part of the hippocampal formation, represents a primary target region of neural degeneration in amnestic mild cognitive impairment $(\mathrm{aMCl})$. Previous studies have revealed subtle structural deficits of the CA subregions (CA1-CA3, bilateral) in aMCl; however, it is not clear how the network function is impacted by aMCl. The present study examined longitudinal changes in resting state functional connectivity (FC) of each CA subregion and how these changes relate to neuropsychological profiles in aMCl.

Methods: Twenty aMCl and 20 healthy control $(\mathrm{HC})$ participants underwent longitudinal cognitive assessment and resting state functional MRI scans at baseline and 15 months afterward. Imaging data were processed with published routines in SPM8 and CONN software. Two-way analysis of covariance was performed with covariates of age, gender, education level, follow up interval, gray matter volume, mean FD, as well as global correlation (GCOR). Pearson's correlation was conducted to evaluate the relationship between the longitudinal changes in CA subregional FC and neuropsychological performance in aMCl subjects.

Results: Resting state FC between the right CA1 and right middle temporal gyrus (MTG) as well as between the left CA2 and bilateral cuneal cortex (CC) were decreased in aMCl subjects as compared to $\mathrm{HC}$. Longitudinal decrease in FC between the right CA1 and right MTG was correlated with reduced capacity of episodic memory in aMCl subjects.

Conclusion: The current findings suggest functional alterations in the CA subregions. CA1 connectivity with the middle temporal cortex may represent an important neural marker of memory dysfunction in aMCl.

Keywords: cornu ammonis, hippocampus, amnestic mild cognitive impairment, functional connectivity, longitudinal study 


\section{INTRODUCTION}

Amnestic mild cognitive impairment (aMCI) precedes the onset of Alzheimer's disease (AD) and comprises a syndrome of cognitive decline without notable interference with daily activities (Gauthier et al., 2006; Petersen, 2011). The hippocampus plays a critical role in the encoding, consolidation, storage, and retrieval of episodic memories (Ranganath et al., 2005; Okada and Okaichi, 2009; Langston et al., 2010). Abundant evidence has suggested that the hippocampus is one of the most specific and sensitive pathological sites of AD (Ulrich, 1985; Delacourte et al., 1999; Chetelat and Baron, 2003; Maruszak and Thuret, 2014).

The hippocampus is divided into fascia dentate (dentate gyrus or DG), subregions of the cornu ammonis (CA1-CA4), and subiculum (SUB). Using in vivo magnetic resonance imaging (MRI), architectonic maps of the CA1-CA3, the subicular complex, fascia dentata together with CA4, and the hippocampalamygdaloid transition area have been generated (Amunts et al., 2005). Neuropathological studies revealed the progression of neurodegeneration throughout the hippocampus (Gomez-Isla et al., 1996; Schonheit and Zarski, 2004) and indicated that CA1 might be the first hippocampal subregion being affected by neurofibrillary changes and neuronal loss (Braak and Braak, 1991; Braak et al., 1993). Structural MRI studies demonstrated differential involvement of the hippocampal subfields in normal aging, MCI, AD, and semantic dementia (La Joie et al., 2010; de Flores et al., 2015). The patterns of volumetric reduction suggested most severe atrophy in the CA1, followed by SUB, $\mathrm{CA} 2$, and $\mathrm{CA} 3 / \mathrm{DG}$ in $\mathrm{MCI}$ and $\mathrm{AD}$ patients, in association with cognitive deterioration (Apostolova et al., 2010; La Joie et al., 2013; Tang et al., 2015). These findings indicate that although structural deficits involve all of the hippocampal subregions, the onset time and severity may vary across them.

In contrast to structural MRI showing the anatomical characteristics, functional MRI measures brain activity by detecting changes in blood oxygenation level dependent (BOLD) signals. It has been proposed that functional alterations likely precede structural changes (Greicius et al., 2004; Fox and Raichle, 2007). Low-frequency fluctuation of the BOLD signals, as revealed by resting state functional MRI, provides a viable approach to assess the network functional integrity of structurally segregated brain regions. A previous resting state functional MRI work demonstrated dysconnectivity of the hippocampal subfields in aMCI subjects. Specifically, functional connectivity (FC) decreased between the SUB and posterior cingulate cortex (PCC), right ventromedial prefrontal cortex, as well as left superior frontal cortex in aMCI as compared to health control (HC) participants (de Flores et al., 2017). In addition, a longitudinal resting state functional MRI study demonstrated that aMCI subjects exhibited changes in FC of bilateral CA, SUB, and DG with the PCC, lateral temporal cortex, medial frontal gyrus, insula, and cerebellum over time (Bai et al., 2011).

However, the aforementioned studies did not distinguish the CA1-CA3 subregions. Previous studies have revealed that different CA subregions harbor distinct functions in learning and memory (Tsien et al., 1996; Nakashiba et al., 2008;
Coras et al., 2014; Adnan et al., 2016; Sormaz et al., 2017; Shah et al., 2018). The CA1 has been considered as a mismatch detector of environmental inputs versus stored representations, as well as a contextual filter to associate temporally discontiguous stimuli (Wallenstein et al., 1998; Rolls and Kesner, 2006). In addition, along with $\mathrm{CA} 2$, CA1 contributes to the associative memory deficits seen in MCI subjects (Atienza et al., 2011). Investigated only very recently, the CA2 has been implicated in memory consolidation (Oliva et al., 2016), social memory (Hitti and Siegelbaum, 2014), and especially in spatial memory (Alexander et al., 2016). Lastly, the CA3 plays an important role in associating the stimuli-matched patterns with previous experiences (Nakazawa et al., 2002; Nakashiba et al., 2008). Thus, it would be important to examine how CA subregional resting state FC may be altered in aMCI, as compared to HC, crosssectionally and longitudinally.

In the present study, we investigated whole-brain FC in individuals with aMCI and demographically matched $\mathrm{HC}$, both at baseline and after 15 months of follow-up. On the basis of previous studies, we hypothesized that, in comparison with HC, aMCI subjects might demonstrate CA subregion-specific changes in FC with cortical and subcortical regions and these changes might aggravate as the illness progresses. Further, we explored how these functional brain changes relate to neuropsychological deficits in aMCI.

\section{MATERIALS AND METHODS}

\section{Participants}

The study was conducted under a research protocol approved by the Ethics Committee of the Xuanwu Hospital. A written informed consent was obtained from all participants prior to the study.

Twenty aMCI subjects and $20 \mathrm{HC}$ participants were recruited from the Xuanwu Hospital of Capital Medical University in Beijing. At baseline, all the participants underwent detailed evaluation of medical history, complete physical examination, MRI scan, and neuropsychological evaluation with Mini Mental State Examination (MMSE), Clinical Dementia Rating (CDR), Montreal Cognitive Assessment (MoCA), and Auditory Verbal Learning Test (AVLT). After 15 months, all the participants completed a follow-up MRI scan and neuropsychological evaluation. MRI parameters and neuropsychological tests at follow-up were identical to those undertaken at baseline.

The diagnosis of aMCI followed the criteria stipulated by the National Institute on Aging and the Alzheimer's Association (Albert et al., 2011), which include: (a) complaint of cognitive change; (b) cognitive function impairment, especially in episodic memory; (c) ability to maintain independence in daily activities; (d) not demented; (e) CDR-SB (Sum of the Boxes) score $=0.5$, with a score of at least 0.5 on the memory domain (Petersen et al., 2001); and (f) a score $>1$ of visual rating of medial temporal lobe atrophy on coronal Tl-weighted MRI (Scheltens et al., 1992).

The criteria for the HC were as follows: (a) no complaints of cognitive changes; (b) absence of abnormal findings in brain MRI; (c) no neurological deficiencies in physical examinations; (d) no 
current or previous diagnosis of any neurological or psychiatric disorders; and (e) CDR-SB score $=0$.

Participants were excluded from the study if they had a previous diagnosis of stroke, head injury, alcohol use disorder, epilepsy, depression, Parkinson's disease or other neurological or psychiatric illness, major medical illness, and severe visual or hearing loss. Additional exclusion criteria for both aMCI and HC participants included contraindications for MRI, such as the use of cardiac pacemakers and claustrophobia.

\section{Magnetic Resonance Imaging Procedures}

The subjects were scanned using a 3-Tesla Trio scanner (Siemens, Erlangen, Germany). All the participants were asked to hold still, keep their eyes closed, and stay awake. Foam pads were employed to minimize head motion and headphones were used to reduce scanner noise. Resting state functional MRI images were acquired using an echo-planar imaging (EPI) sequence: repetition time $(\mathrm{TR})=2000 \mathrm{~ms}$, echo time $(\mathrm{TE})=40 \mathrm{~ms}$, flip angle $(\mathrm{FA})=90^{\circ}$, field of view $(\mathrm{FOV})=256 \mathrm{~mm}, 28$ axial slices, slice thickness $/$ gap $=4 / 1 \mathrm{~mm}$, bandwidth $=2232 \mathrm{~Hz} /$ pixel, and number of repetitions $=239$. The $3 \mathrm{D} \mathrm{T} 1$-weighted anatomical images were acquired with a magnetization-prepared rapid gradient echo (MPRAGE) method with the following parameters: $\mathrm{TR}=1900 \mathrm{~ms}, \mathrm{TE}=2.2 \mathrm{~ms}$, inversion time $(\mathrm{TI})=900 \mathrm{~ms}, \mathrm{FA}=9^{\circ}$, bandwidth $=199 \mathrm{~mm}$, matrix $=256 \times 224,176$ sagittal slices, and slice thickness $=1 \mathrm{~mm}$.

\section{MRI Data Preprocessing}

Resting state functional MRI images were preprocessed using Statistical Parametric Mapping software SPM8 ${ }^{1}$, and seed-tovoxel correlation analysis was carried out by the functional connectivity (CONN) toolbox v17c (Whitfield-Gabrieli and Nieto-Castanon, 2012). The first 10 images of each resting state functional MRI data set were discarded to reduce the initial fluctuation of MRI signals. Images of each individual subject were corrected for slice timing and realigned. Participants with head motion more than $1.5 \mathrm{~mm}$ in translation or more than $1.5^{\circ}$ in rotation were excluded. The resulting images were normalized to the Montreal Neurological Institute (MNI) space and resampled to $2 \mathrm{~mm} \times 2 \mathrm{~mm} \times 2 \mathrm{~mm}$. Subsequently, the functional images were smoothed with a $4-\mathrm{mm}$ full width at half maximum (FWHM) isotropic Gaussian kernel. After preprocessing, images were then band-pass filtered to $0.008 \sim 0.09 \mathrm{~Hz}$ to reduce the influence of noise. Further denoising involved regression of the six motion parameters and their first-order derivatives, regression of white matter and cerebrospinal fluid signals (Behzadi et al., 2007) and linear detrending.

\section{Gray Matter Volume Analysis}

The six CA seeds (right- and left- hemispheric CA1, CA2, and CA3) and bilateral hippocampi were defined through the Anatomy Toolbox in SPM8 (Eickhoff et al., 2005). Structural data processing was performed using SPM8 including normalization

${ }^{1}$ https://www.fil.ion.ucl.ac.uk/spm into the standard MNI apace, segmentation, modulation, and smoothing with an 8-mm full-width at half-maximum Gaussian kernel. The gray matter volumes of bilateral hippocampi and the six CA-subfields were then extracted based on the smoothed modulated gray matter maps and the latter was included in data analyses as nuisance covariates to rule out the influence of volumetric differences on FC.

\section{Functional Connectivity Analysis}

For individual subjects, a mean time series each for the six CA seeds was computed as the reference time course. The Pearson correlation coefficients between the seed and all other brain voxels were computed to generate correlation maps. For group analyses, the correlation coefficients were converted to $\mathrm{z}$-value using Fisher's r-to-z transformation to improve normality (Lowe et al., 1998).

\section{Statistical Analysis}

Demographic data and neuropsychological measures were analyzed using SPSS 19 . Student $t$-tests were applied to compare continuous variables, including age, years of education, and neuropsychological scores, and a chi-square test was applied to examine the differences in gender composition between groups.

In the random effects analyses of imaging data, we first determined the patterns of voxelwise CA subregional FC within each group, using one sample $t$-tests without covariates, and evaluated the results at cluster level $p<0.05$ corrected for multiple comparisons using the family-wise error rate (FWE). We then employed a 2 (time: follow-up versus baseline) $\times 2$ (group: aMCI versus HC) flexible factorial design, with four conditions aMCI baseline (aMCI1), aMCI follow-up (aMCI2), HC baseline ( $\mathrm{HC} 1$ ), and $\mathrm{HC}$ follow-up (HC2), to examine time and group main effects as well as time by group interaction effect. Results were thresholded at cluster level $p<0.05$, FWE corrected. The covariates used in between-group analyses included age, gender, years of education, follow-up interval, gray matter volume, mean FD, as well as global correlation (GCOR) index. The GCOR index represented the average correlation coefficient between every pair of voxels across the entire brain, a measure of network centrality at each voxel computed for each subject during the denoising step in CONN (Saad et al., 2013). Brain regions were localized with software xjView ${ }^{2}$.

The group main effects were examined by $[(\mathrm{HC} 1+\mathrm{HC} 2)>(\mathrm{aMCI} 1+\mathrm{aMCI} 2)]$ and $[(\mathrm{aMCI} 1+\mathrm{aMCI} 2)>(\mathrm{HC} 1+\mathrm{HC} 2)]$ to determine decreased and increased FC in aMCI compared to $\mathrm{HC}$ participants for each CA subfield, respectively. The time main effects were evaluated by $[(\mathrm{aMCI} 1+\mathrm{HC} 1)>(\mathrm{aMCI} 2+\mathrm{HC} 2)]$ and $[(\mathrm{aMCI} 2+\mathrm{HC} 2)>(\mathrm{aMCI} 1+\mathrm{HC} 1)]$ to determine decreased and increased FC at follow-up compared to baseline, respectively. The time by group interaction effects were evaluated by $[(\mathrm{aMCI} 2>\mathrm{aMCI} 1)>(\mathrm{HC} 2>\mathrm{HC} 1)]$ to identify increased FC of CA subregions in aMCI relative to $\mathrm{HC}$ and $[($ aMCI1 $>$ aMCI2 $)>(\mathrm{HC} 1>\mathrm{HC} 2)]$ to identify decreased FC of $\mathrm{CA}$ subregions in aMCI relative to $\mathrm{HC}$.

\footnotetext{
${ }^{2}$ http://www.alivelearn.net/xjview
} 
Post hoc pairwise comparisons were conducted focusing on the CA subregions that showed significant main and interaction effects. Independent-samples $t$-tests were applied to compute the group differences of FC between $\mathrm{HC}$ and aMCI at baseline and follow-up separately. Decreased FC in aMCI group was determined by $(\mathrm{HC} 1>$ aMCI1) and $(\mathrm{HC} 2>$ aMCI2) while increased FC in aMCI group was determined by (aMCI1 > HC1) and $($ aMCI2 $>$ HC2). Paired-samples $t$-tests were performed to explore the longitudinal FC changes within each group with $(\mathrm{aMCI} 1>\mathrm{aMCI} 2)$ and $(\mathrm{HC} 1>\mathrm{HC} 2)$ to identify decreased FC at follow-up and (aMCI2 > aMCI1) and (HC2 > HC1) to identify increased FC at follow-up, as compared to baseline.

\section{Correlation Analysis}

Regions-of-interest (ROI) were defined based on the regions showing significant FC changes in CA subregions in aMCI compared to $\mathrm{HC}$ in the interaction effect analysis. For each subject the mean FC values across all voxels of each ROI was computed. Pearson correlation analysis was then conducted to evaluate the relationship between the longitudinal changes of the FC and longitudinal changes of neuropsychological data in aMCI subjects. Statistical significance was set at $p<0.05$, Bonferroni corrected for multiple comparisons.

\section{RESULTS}

\section{Demographics and Neuropsychological Assessment}

As shown in Table 1, aMCI and HC participants did not differ in gender, age, and education level while significant volumetric difference of the two groups in bilateral hippocampi were found. Compared to HC, aMCI group showed significant general cognitive decline as reflected by the scores in the MMSE $(p<0.001)$, MoCA $(p<0.001)$, and CDR $(p<0.001)$, as well as poorer memory performance as reflected by the AVLT immediate and delayed recall and recognition scores $(p<0.001$ for all comparisons), both at baseline and follow-up. Compared to baseline, the subjects with aMCI showed worse cognitive performance in the MMSE $(p=0.017)$ and MoCA $(p=0.048)$ at follow-up, confirming more severe memory impairment as the disease progressed. Besides, two aMCI subjects developed mild $\mathrm{AD}$ while the others and all of the $\mathrm{HC}$ remained stable.

\section{CA Subregional FC: Within-Group Analysis}

In $\mathrm{HC}$ group, both at baseline and follow-up, significant connectivities with the medial temporal lobe, including the hippocampus, parahippocampus, and amygdala, were identified for each of the six CA subregions (Supplementary Figures 1-6). Bilateral CA1 showed additional connectivities with bilateral superior/middle/inferior temporal gyri (Supplementary Figures 1, 2). In aMCI group, both at baseline and follow-up, FC patterns similar to HC group were revealed with relatively smaller cluster sizes (Supplementary Figures 1-6).

\section{CA Subregional FC: ANCOVA}

The main effect of "group" revealed significantly decreased FC changes between right CA1 (CA1R) and right middle temporal gyrus (MTG), as well as left PCC in aMCI in contrast to HC. The main effect of "time" revealed decreased CA1R-FC with the right MTG and decreased CA2L-FC with bilateral cuneal cortex (CC) for follow-up in contrast to baseline.

In addition, we explored the differences of longitudinal changes between aMCI and HC group in "group" by "time" interaction effect. The CA1R-FC showed greater decreases with the right MTG and CA2L-FC showed greater decreases with bilateral CC across time in aMCI in contrast to HC (Table 2 and Figures 1A,B).

Post hoc pairwise comparisons showed that: 1: at baseline, no significant CA subregional FC changes were found in aMCI as compared to HC; 2: at follow-up, aMCI subjects demonstrated decreased CA1R-FC with right MTG (Figure 2A), right frontal pole (FP, Figure 2B), and left PCC (Figure 2C), as compared to $\mathrm{HC}$; 3: no significant longitudinal FC changes were observed in aMCI or HC group.

\section{Correlation With Cognitive Measures}

The two regions showing significant time by group interaction the right MTG and bilateral CC - were defined as ROIs and the connectivity strength of each ROI was extracted for correlation analysis. The results showed that longitudinal changes (followup vs. baseline) in FC between the right CA1 and the right MTG were positively correlated with longitudinal changes (follow-up vs. baseline) in the capacity of episodic memory $(r=0.523$, $p=0.018)$, as indexed by the delayed recall score of AVLT (Figure 2D).

\section{DISCUSSION}

We examined the differences in subjects with aMCI and healthy control (HC) participants in CA subregional (CA1-CA3) restingstate FC. Amnestic MCI subjects demonstrated decreased right CA1 (CA1R)-FC with the right MTG and left CA2 (CA2L)FC with bilateral CC, in contrast to HC. Further, longitudinal changes of CA1R-FC with right MTG were associated with longitudinal changes of episodic memory across time. The findings suggest a potential hippocampal neural marker of dementia.

The CA1, an important region of both polysynaptic and monosynaptic pathways in the hippocampal mnemonic circuits, plays a crucial role in memory processing. Previous structural studies have suggested CA1 as the most vulnerable hippocampal subregions to be impacted in dementia (Apostolova et al., 2010; Tang et al., 2015). Morphological analyses revealed that decreases in gray matter volume and cortical thickness frequently occurred in the CA1 subfield in aMCI subjects (Yushkevich et al., 2015). A histopathological study demonstrated a significant association between CA1-specific atrophy and beta-amyloid deposition (Apostolova et al., 2015). Apostolova et al. (2015) also reported a significant association between changes in CA1 
TABLE 1 | Clinical characteristics of subjects with amnestic mild cognitive impairment (aMCl) and healthy control (HC).

\begin{tabular}{|c|c|c|c|c|c|c|}
\hline & \multicolumn{3}{|c|}{ Baseline } & \multicolumn{3}{|c|}{ Follow-up } \\
\hline & $\mathrm{HC}$ & $\mathrm{aMCl}$ & $p$-value & $\mathrm{HC}$ & $\mathrm{aMCl}$ & $p$-value \\
\hline Age (yrs) & $67.25 \pm 7.50$ & $66.95 \pm 9.65$ & 0.91 & $68.67 \pm 7.54$ & $68.10 \pm 9.67$ & 0.84 \\
\hline Gender (m/f) & $10 / 10$ & $7 / 13$ & 0.34 & $10 / 10$ & $7 / 13$ & 0.34 \\
\hline Education (yrs) & $11.90 \pm 3.82$ & $9.65 \pm 4.06$ & 0.08 & $11.90 \pm 3.82$ & $9.65 \pm 4.06$ & 0.08 \\
\hline Volume of Lt. HP & $0.562 \pm 0.016$ & $0.538 \pm 0.026$ & 0.002 & $0.560 \pm 0.017$ & $0.534 \pm 0.027^{\dagger}$ & 0.001 \\
\hline Volume of Rt. HP & $0.538 \pm 0.016$ & $0.521 \pm 0.027$ & 0.020 & $0.535 \pm 0.016^{\dagger}$ & $0.516 \pm 0.029^{\dagger}$ & 0.013 \\
\hline CDR-SB & $0(20)$ & $0.5(20)$ & $<0.001$ & $0(20)$ & $0.5(18) / 1(2)$ & $<0.001$ \\
\hline MMSE & $28.60 \pm 1.23$ & $24.80 \pm 3.70$ & $<0.001$ & $28.35 \pm 1.46$ & $23.45 \pm 3.47^{\dagger}$ & $<0.001$ \\
\hline MoCA & $26.40 \pm 1.63$ & $20.85 \pm 4.70$ & $<0.001$ & $26.50 \pm 1.87$ & $19.50 \pm 4.91^{\dagger}$ & $<0.001$ \\
\hline AVLT-immediate recall & $8.88 \pm 1.63$ & $6.00 \pm 1.45$ & $<0.001$ & $8.95 \pm 1.25$ & $6.08 \pm 1.38$ & $<0.001$ \\
\hline AVLT-delayed recall & $9.35 \pm 3.54$ & $4.55 \pm 2.78$ & $<0.001$ & $9.25 \pm 3.56$ & $4.90 \pm 3.02$ & $<0.001$ \\
\hline AVLT-recognition & $11.25 \pm 3.05$ & $8.45 \pm 3.36$ & $<0.001$ & $11.70 \pm 2.22$ & $7.75 \pm 3.24$ & $<0.001$ \\
\hline
\end{tabular}

Values represent means $\pm S D$; Lt., left; Rt., right; HP, hippocampus; CDR-SB, clinical dementia rate-sum of the boxes; MMSE, mini mental state examination; MoCA,

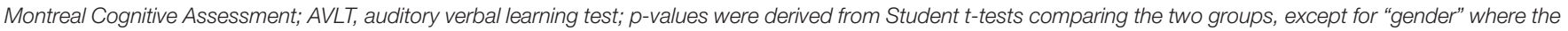
$p$-value was obtained using the $\chi^{2}$-test; ${ }^{\dagger}$ represents significant decline in follow-up vs. baseline.

TABLE 2 | Longitudinal changes in functional connectivity to the cornu ammonis subregions in aMCl subjects as compared to HC.

\begin{tabular}{|c|c|c|c|c|c|c|}
\hline Seed regions & Region & Cluster size (voxel) & & MNI (x y z) & & $F / T$-value \\
\hline \multirow[t]{7}{*}{ CA1R } & $\mathrm{HC}$ vs. aMCl: $[(\mathrm{HC} 1+\mathrm{HC} 2)>(\mathrm{aMCl} 1+\mathrm{aMCl} 2)]$ & & & & & \\
\hline & Lt. PCC & 190 & -6 & -46 & 36 & $12.67^{*}$ \\
\hline & Rt. MTG & 74 & 60 & -48 & 8 & $16.01^{*}$ \\
\hline & Baseline vs. Follow-up: [(aMCl1 + HC1) > (aMCl2 + HC2) $]$ & & & & & \\
\hline & Rt. MTG & 114 & 64 & -48 & 8 & $24.40^{*}$ \\
\hline & {$[(\mathrm{aMCl} 1>\mathrm{aMCl} 2)>(\mathrm{HC} 1>\mathrm{HC} 2)]$} & & & & & \\
\hline & Rt. MTG & 108 & 64 & -48 & 8 & 5.51 \\
\hline \multirow[t]{4}{*}{ CA2L } & 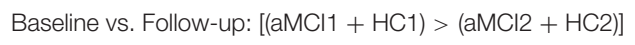 & & & & & \\
\hline & Bi. CC & 66 & -2 & -76 & 24 & $18.95^{*}$ \\
\hline & {$[(\mathrm{aMCl} 1>\mathrm{aMCl} 2)>(\mathrm{HC} 1>\mathrm{HC} 2)]$} & & & & & \\
\hline & Bi. CC & 125 & 0 & -78 & 24 & 5.26 \\
\hline
\end{tabular}

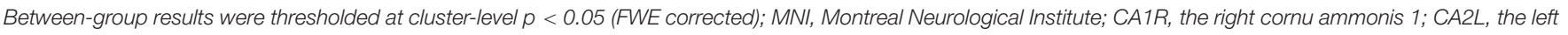
cornu ammonis 2; Lt., left; Rt., right; Bi., bilateral; PCC, posterior cingulate cortex; MTG, middle temporal gyrus; CC, cuneal cortex; *represents F-value.

volume and neuronal count, which might contribute to memory dysfunction. Furthermore, atrophy of the CA1R was significantly associated with conversion to dementia (Apostolova et al., 2010) and appeared to be a reliable predictor of the development of $\mathrm{AD}$ in MCI subjects within a relatively short period of 3 years (Chow et al., 2015). Here, we demonstrated significantly decreased FC of CA1R over a 15-month follow-up period in aMCI subjects. The current results suggested crucial contribution of CA1 dysfunction to cognitive impairment in aMCI.

Consistent with a previous study, no significant CA subregional FC changes were found in aMCI subjects at baseline (de Flores et al., 2017). At follow-up, aMCI subjects as compared with $\mathrm{HC}$ subjects demonstrated significantly decreased FC between the CA1R and right MTG, right FP, as well as left PCC. Further, longitudinal changes in FC between the CA1R and right MTG were positively related to longitudinal changes of episodic memory as measured by delayed recall scores of AVLT, suggesting significant association between disrupted CA1R-FC and progressive cognitive alterations in aMCI. Functionally associated with verbal and visual semantic knowledge (Vandenberghe et al., 1996), the MTG supports verbal short-term memory (Raettig and Kotz, 2008). Previous studies reported that the MTG was one of the key brain regions of tau accumulation in aMCI and AD patients (Maass et al., 2017) and showed significant volume reduction during the disease process (Cha et al., 2013; Li K. et al., 2017). Further, abnormal FC between MTG and other brain regions, such as hypothalamus (Liu et al., 2018) and default mode network (Li M. et al., 2017), contributed to the cognitive impairment in aMCI and $\mathrm{AD}$. In line with the aforementioned studies, decreases in the FC between CA1R and the right MTG may underline the role of the MTG in the clinical manifestation of aMCI and development of AD.

The CC, most known for its involvement in basic visual processing, is a key region of the occipito-parieto-frontal network in support of spatial information processing (Gomez et al., 2014). Active visual experiences induce visual cortex synaptic plasticity and this phenomenon can influence the following spatial episodes integration in hippocampus (Debanne, 1996; Tsanov and Manahan-Vaughan, 2008). Recent studies have implicated the CA2 in spatial information encoding and storage. 
A
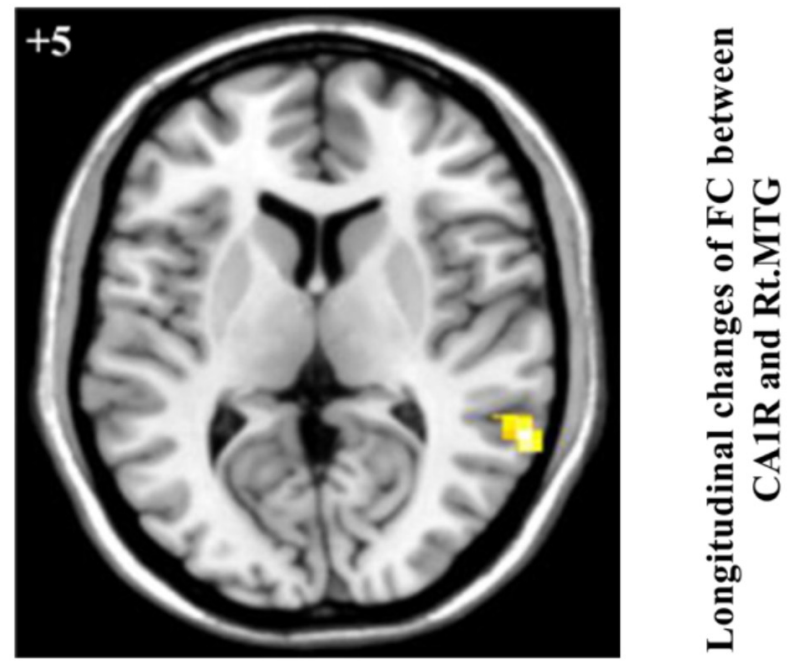

B

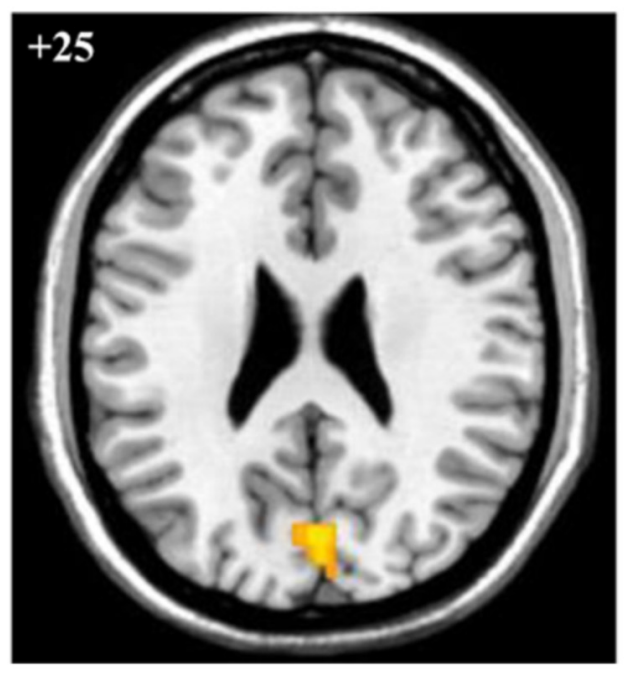

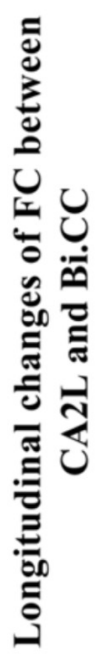
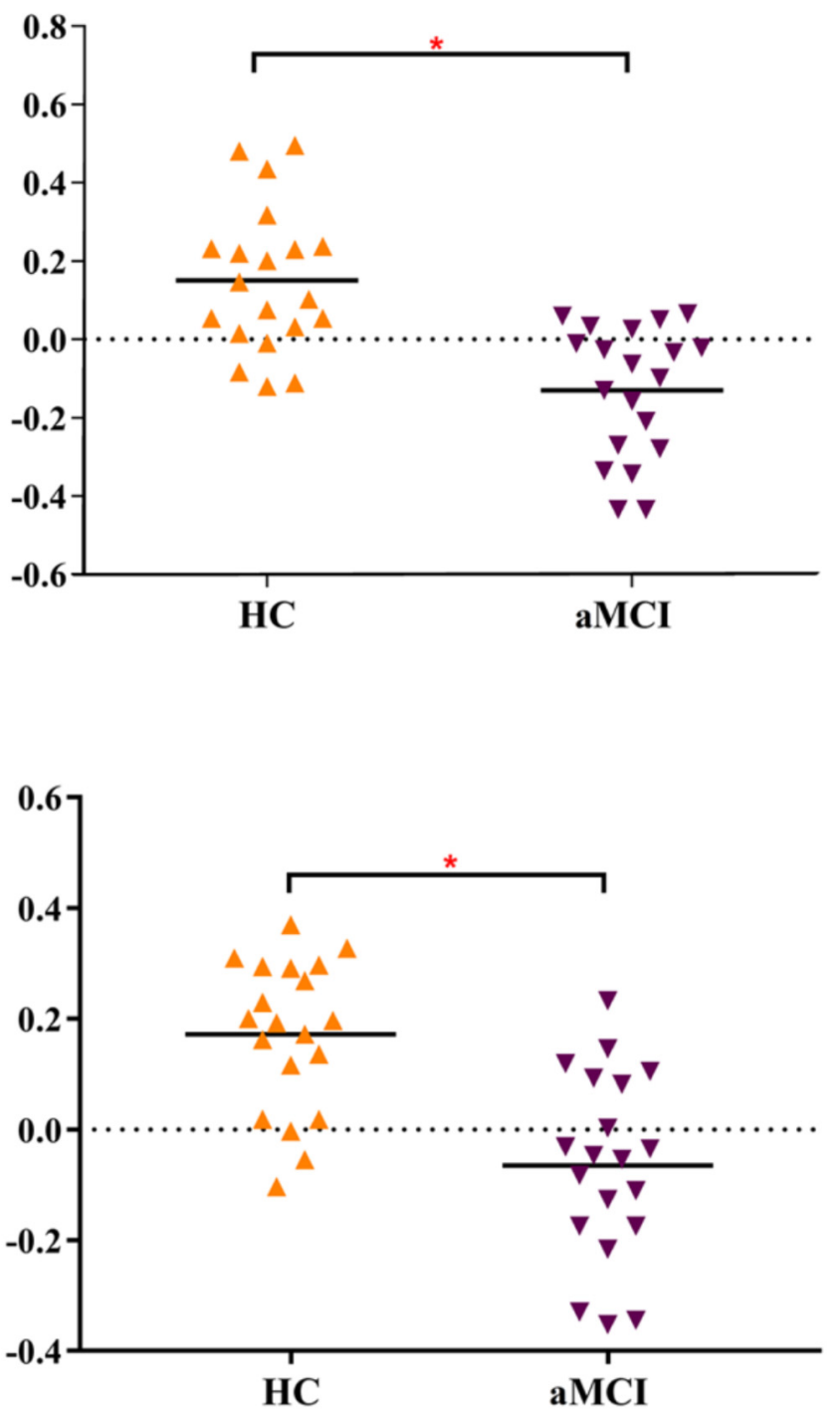

FIGURE 1 | (A) Decreased FC between right CA1 and right MTG in aMCl subjects as compared to HC at follow-up vs. baseline (interaction effect); (B) Decreased FC between left $\mathrm{CA} 2$ and bilateral $\mathrm{CC}$ in aMCl subjects as compared to $\mathrm{HC}$ group at follow-up vs. baseline (interaction effect). Numbers in the figure indicate the $Z$ coordinate in MNI space; FC, functional connectivity; CA1R, the right cornu ammonis 1; CA2L, the left cornu ammonis 1; Rt., right; Bi., bilateral; MTG, middle temporal gyrus; CC, cuneal cortex; *indicates cluster level $p<0.05$, FWE corrected.

Alexander et al. (2016) showed that the ability to modify the existing spatial representations by globally remapping the place fields was unique to neurons in the CA2. Moreover, CA2 neurons form a highly plastic disynaptic circuit to mediate key aspects of hippocampal-dependent spatial memory by integrating inputs from the entorhinal cortex and outputs to the CA1 (Chevaleyre and Siegelbaum, 2010). In the studies related with human hippocampal asymmetry, it has been suggested that, while the right hippocampus is related with an allocentric spatial representation concerning places, the left hippocampus significantly activates when using a sequential egocentric spatial representation, which likely associates with the spatiotemporal aspect of episodic memory (Igloi et al., 2010; Shipton et al., 2014; El-Gaby et al., 2015). In the present study, subjects with aMCI demonstrated decreased CA2L-FC with bilateral cuneal cortices. It is possible that the disrupted CA2L-FC may contribute to the spatial components of episodic memory impairment during the progression of cognitive impairment. The functional implications of this finding remain to be investigated in the future.

Several limitations need to be considered for this study. First, the mean follow-up intervals in aMCI and $\mathrm{HC}$ were group matched but there were inter-subject variations. Although this factor has been included as a nuisance covariate in data analyses, we cannot entirely rule out the influence of follow-up interval 


\section{A}

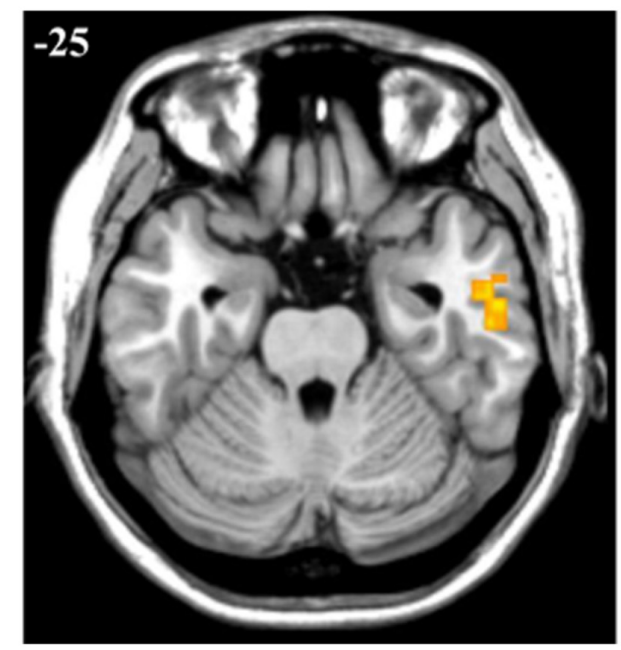

c

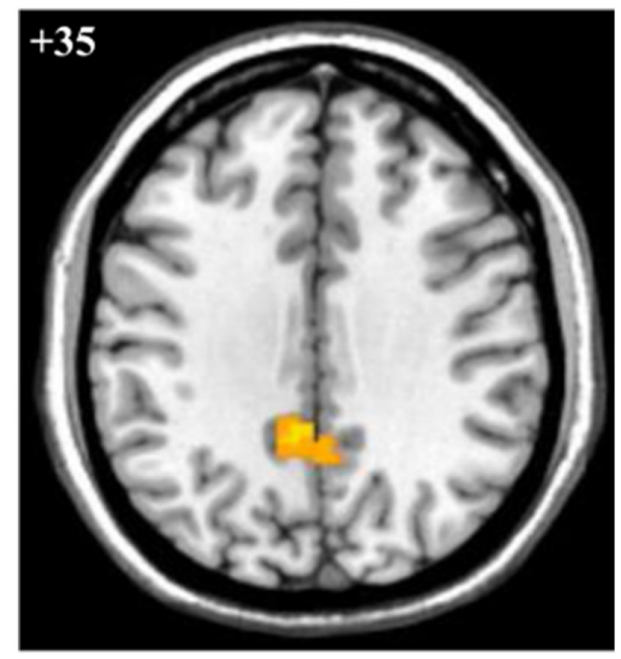

B

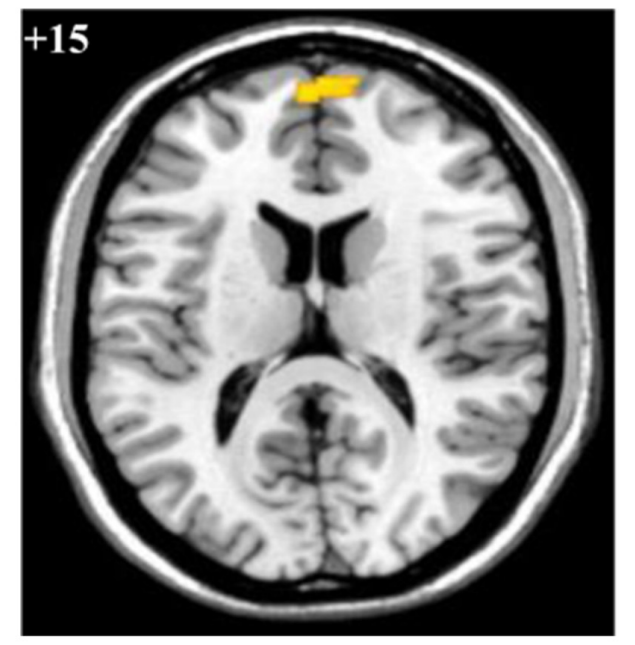

D

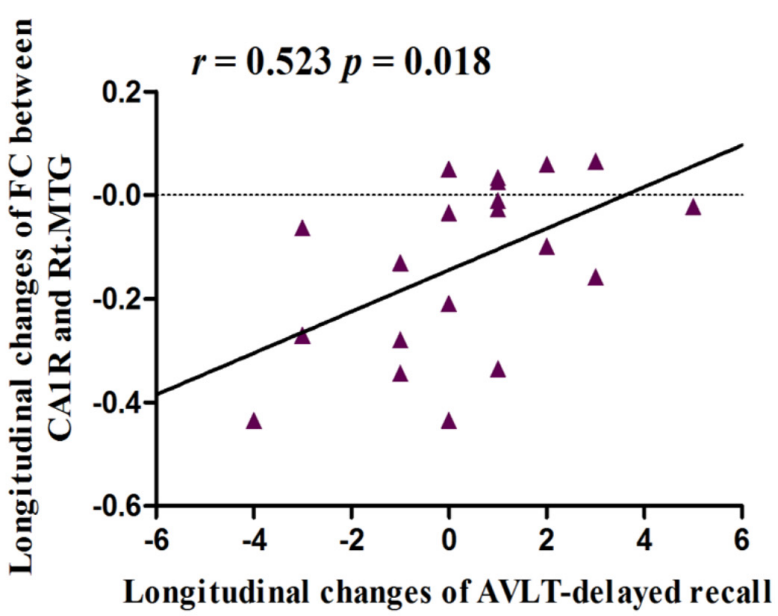

FIGURE 2 | (A) Decreased FC between right CA1 and right MTG in aMCl subjects as compared to HC group at follow-up; (B) Decreased FC between right CA1 and right $\mathrm{FP}$ in aMCl subjects as compared to $\mathrm{HC}$ group at follow-up; (C) Decreased FC between right $\mathrm{CA} 1$ and left PCC in aMCl subjects as compared to HC group at follow-up; (D) Linear correlation of longitudinal changes in FC between right CA1 and right MTG and longitudinal changes of AVLT-delayed recall scores in aMCl subjects. Numbers in the figure indicate the $Z$ coordinate in MNI space; FC, functional connectivity; CA1R, the right cornu ammonis 1; Lt., left; Rt., right; MTG, middle temporal gyrus; FP, frontal pole; PCC, posterior cingulate cortex; AVLT, Auditory Verbal Learning Test.

on the current results. Second, the sample size of the study was relatively small, and studies with a larger sample will be needed to replicate the current findings and to elucidate CA subregional functions and dysfunctions in aMCI. Third, without access to PET examination and cerebrospinal fluid analysis, the criteria used in the present study indicated an intermediate likelihood that the MCI syndrome is due to $\mathrm{AD}$. The recently proposed $\mathrm{A}$ (beta-amyloid) - T (tau) - N (neuro-degeneration) system should be referred to in the future study (Jack et al., 2018).

In summary, we identified and dissociated FC alterations for CA subfields in subjects with aMCI across time. Specifically, functional dysconnectivity in the CA1R was prominently affected in aMCI, in association with cognitive decline, at 15month follow-up. These new findings add to the literature of hippocampal dysfunction and may facilitate future research of neural markers of cognitive decline in aMCI and AD.

\section{AUTHOR CONTRIBUTIONS}

HL and XJ carried out data collection and analysis and wrote the manuscript. ZQ and JL helped with data interpretation. XF, TM, $\mathrm{RP}$, and HN carried out data collection. C-sL contributed to the conceptualization of the study and revision of the manuscript. 
KL contributed to the conceptualization and design of the study and revised the manuscript.

\section{FUNDING}

This work was supported partly by grants from the National Natural Science Foundation of China (81471649, 81571648, and 81370037), Clinical Medicine Development Special Funding from the Beijing Municipal Administration of Hospital (ZYLX201609), Key Projects in the National Science and

\section{REFERENCES}

Adnan, A., Barnett, A., Moayedi, M., Mccormick, C., Cohn, M., and Mcandrews, M. P. (2016). Distinct hippocampal functional networks revealed by tractography-based parcellation. Brain Struc. Funct. 221, 2999-3012. doi: 10. 1007/s00429-015-1084-x

Albert, M. S., Dekosky, S. T., Dickson, D., Dubois, B., Feldman, H. H., Fox, N. C., et al. (2011). The diagnosis of mild cognitive impairment due to Alzheimer's disease: recommendations from the National Institute on AgingAlzheimer's Association workgroups on diagnostic guidelines for Alzheimer's disease. Alzheimers Dement. 7, 270-279. doi: 10.1016/j.jalz.2011.03.008

Alexander, G. M., Farris, S., Pirone, J. R., Zheng, C., Colgin, L. L., and Dudek, S. M. (2016). Social and novel contexts modify hippocampal CA2 representations of space. Nat. Commun. 7:10300. doi: 10.1038/ncomms10300

Amunts, K., Kedo, O., Kindler, M., Pieperhoff, P., Mohlberg, H., Shah, N. J., et al. (2005). Cytoarchitectonic mapping of the human amygdala, hippocampal region and entorhinal cortex: intersubject variability and probability maps. Anat. Embryol. 210, 343-352. doi: 10.1007/s00429-005-0025-5

Apostolova, L. G., Thompson, P. M., Green, A. E., Hwang, K. S., Zoumalan, C., Jack, C. J., et al. (2010). 3D comparison of low, intermediate, and advanced hippocampal atrophy in MCI. Hum. Brain Mapp. 31, 786-797. doi: 10.1002/ hbm.20905

Apostolova, L. G., Zarow, C., Biado, K., Hurtz, S., Boccardi, M., Somme, J., et al. (2015). Relationship between hippocampal atrophy and neuropathology markers: a 7T MRI validation study of the EADC-ADNI Harmonized Hippocampal Segmentation Protocol. Alzheimers Dement. 11, 139-150. doi: 10.1016/j.jalz.2015.01.001

Atienza, M., Atalaia-Silva, K. C., Gonzalez-Escamilla, G., Gil-Neciga, E., SuarezGonzalez, A., and Cantero, J. L. (2011). Associative memory deficits in mild cognitive impairment: the role of hippocampal formation. Neuroimage 57, 1331-1342. doi: 10.1016/j.neuroimage.2011.05.047

Bai, F., Xie, C., Watson, D. R., Shi, Y., Yuan, Y., Wang, Y., et al. (2011). Aberrant hippocampal subregion networks associated with the classifications of aMCI subjects: a longitudinal resting-state study. PLoS One 6:e29288. doi: 10.1371/ journal.pone.0029288

Behzadi, Y., Restom, K., Liau, J., and Liu, T. T. (2007). A component based noise correction method (CompCor) for BOLD and perfusion based fMRI. Neuroimage 37, 90-101. doi: 10.1016/j.neuroimage.2007. 04.042

Braak, H., and Braak, E. (1991). Neuropathological stageing of Alzheimer-related changes. Acta Neuropathol. 82, 239-259. doi: 10.1007/BF00308809

Braak, H., Braak, E., and Bohl, J. (1993). Staging of Alzheimer-related cortical destruction. Eur. Neurol. 33, 403-408. doi: 10.1159/000116984

Cha, J., Jo, H. J., Kim, H. J., Seo, S. W., Kim, H. S., Yoon, U., et al. (2013). Functional alteration patterns of default mode networks: comparisons of normal aging, amnestic mild cognitive impairment and Alzheimer's disease. Eur. J. Neurosci. 37, 1916-1924. doi: 10.1111/ejn.12177

Chetelat, G., and Baron, J. C. (2003). Early diagnosis of Alzheimer's disease: contribution of structural neuroimaging. Neuroimage 18, 525-541. doi: 10. 1016/S1053-8119(02)00026-5

Chevaleyre, V., and Siegelbaum, S. A. (2010). Strong CA2 pyramidal neuron synapses define a powerful disynaptic cortico-hippocampal loop. Neuron 66, 560-572. doi: 10.1016/j.neuron.2010.04.013
Technology Pillar Program during the Twelfth Five-year Plan Period (2012BAI10B04) and Beijing Municipal Science and Technology Commission (Z171100000117001), as well as NIH (United States) grant R21MH113134.

\section{SUPPLEMENTARY MATERIAL}

The Supplementary Material for this article can be found online at: https://www.frontiersin.org/articles/10.3389/fnhum. 2018.00413/full\#supplementary-material

Chow, N., Hwang, K. S., Hurtz, S., Green, A. E., Somme, J. H., Thompson, P. M., et al. (2015). Comparing 3T and 1.5T MRI for mapping hippocampal atrophy in the Alzheimer's Disease Neuroimaging Initiative. AJNR Am. J. Neuroradiol. 36, 653-660. doi: 10.3174/ajnr.A4228

Coras, R., Pauli, E., Li, J., Schwarz, M., Rossler, K., Buchfelder, M., et al. (2014). Differential influence of hippocampal subfields to memory formation: insights from patients with temporal lobe epilepsy. Brain 137, 1945-1957. doi: 10.1093/ brain/awu100

de Flores, R., La Joie, R., Landeau, B., Perrotin, A., Mezenge, F., de La Sayette, V., et al. (2015). Effects of age and Alzheimer's disease on hippocampal subfields: comparison between manual and FreeSurfer volumetry. Hum. Brain Mapp. 36, 463-474. doi: 10.1002/hbm.22640

de Flores, R., Mutlu, J., Bejanin, A., Gonneaud, J., Landeau, B., Tomadesso, C., et al. (2017). Intrinsic connectivity of hippocampal subfields in normal elderly and mild cognitive impairment patients. Hum. Brain Mapp. 38, 4922-4932. doi: $10.1002 / \mathrm{hbm} .23704$

Debanne, D. (1996). Associative synaptic plasticity in hippocampus and visual cortex: cellular mechanisms and functional implications. Rev. Neurosci. 7, 29-46. doi: 10.1515/REVNEURO.1996.7.1.29

Delacourte, A., David, J. P., Sergeant, N., Buee, L., Wattez, A., Vermersch, P., et al. (1999). The biochemical pathway of neurofibrillary degeneration in aging and Alzheimer's disease. Neurology 52, 1158-1165. doi: 10.1212/WNL.52.6.1158

Eickhoff, S. B., Stephan, K. E., Mohlberg, H., Grefkes, C., Fink, G. R., Amunts, K., et al. (2005). A new SPM toolbox for combining probabilistic cytoarchitectonic maps and functional imaging data. Neuroimage 25, 1325-1335. doi: 10.1016/j. neuroimage.2004.12.034

El-Gaby, M., Shipton, O. A., and Paulsen, O. (2015). Synaptic plasticity and memory: new insights from hippocampal left-right asymmetries. Neuroscientist 21, 490-502. doi: 10.1177/1073858414550658

Fox, M. D., and Raichle, M. E. (2007). Spontaneous fluctuations in brain activity observed with functional magnetic resonance imaging. Nat. Rev. Neurosci. 8, 700-711. doi: 10.1038/nrn2201

Gauthier, S., Reisberg, B., Zaudig, M., Petersen, R. C., Ritchie, K., Broich, K., et al. (2006). Mild cognitive impairment. Lancet 367, 1262-1270. doi: 10.1016/S01406736(06)68542-5

Gomez, A., Cerles, M., Rousset, S., Remy, C., and Baciu, M. (2014). Differential hippocampal and retrosplenial involvement in egocentric-updating, rotation, and allocentric processing during online spatial encoding: an fMRI study. Front. Hum. Neurosci. 8:150. doi: 10.3389/fnhum.2014.00150

Gomez-Isla, T., Price, J. L., Mckeel, D. J., Morris, J. C., Growdon, J. H., and Hyman, B. T. (1996). Profound loss of layer II entorhinal cortex neurons occurs in very mild Alzheimer's disease. J. Neurosci. 16, 4491-4500. doi: 10.1523/JNEUROSCI. 16-14-04491.1996

Greicius, M. D., Srivastava, G., Reiss, A. L., and Menon, V. (2004). Defaultmode network activity distinguishes Alzheimer's disease from healthy aging: evidence from functional MRI. Proc. Natl. Acad. Sci. U.S.A. 101, 4637-4642. doi: $10.1073 /$ pnas.0308627101

Hitti, F. L., and Siegelbaum, S. A. (2014). The hippocampal CA2 region is essential for social memory. Nature 508, 88-92. doi: 10.1038/nature13028

Igloi, K., Doeller, C. F., Berthoz, A., Rondi-Reig, L., and Burgess, N. (2010). Lateralized human hippocampal activity predicts navigation based on sequence or place memory. Proc. Natl. Acad. Sci. U.S.A. 107, 14466-14471. doi: 10.1073/ pnas. 1004243107 
Jack, C. R., Bennett, D. A., Blennow, K., Carrillo, M. C., Dunn, B., Haeberlein, S. B., et al. (2018). NIA-AA Research Framework: toward a biological definition of Alzheimer's disease. Alzheimers Dement. 14, 535-562. doi: 10.1016/j.jalz.2018. 02.018

La Joie, R., Fouquet, M., Mezenge, F., Landeau, B., Villain, N., Mevel, K., et al. (2010). Differential effect of age on hippocampal subfields assessed using a new high-resolution 3T MR sequence. Neuroimage 53, 506-514. doi: 10.1016/j. neuroimage.2010.06.024

La Joie, R., Perrotin, A., de La Sayette, V., Egret, S., Doeuvre, L., Belliard, S., et al. (2013). Hippocampal subfield volumetry in mild cognitive impairment, Alzheimer's disease and semantic dementia. Neuroimage Clin. 3, 155-162. doi: 10.1016/j.nicl.2013.08.007

Langston, R. F., Stevenson, C. H., Wilson, C. L., Saunders, I., and Wood, E. R. (2010). The role of hippocampal subregions in memory for stimulus associations. Behav. Brain Res. 215, 275-291. doi: 10.1016/j.bbr.2010.07.006

Li, K., Chan, W., Doody, R. S., Quinn, J., and Luo, S. (2017). Prediction of conversion to Alzheimer's Disease with longitudinal measures and time-toevent data. J. Alzheimers Dis. 58, 361-371. doi: 10.3233/JAD-161201

Li, M., Zheng, G., Zheng, Y., Xiong, Z., Xia, R., Zhou, W., et al. (2017). Alterations in resting-state functional connectivity of the default mode network in amnestic mild cognitive impairment: an fMRI study. BMC Med. Imaging 17:48. doi: 10.1186/s12880-017-0221-9

Liu, X., Chen, W., Tu, Y., Hou, H., Huang, X., Chen, X., et al. (2018). The abnormal functional connectivity between the hypothalamus and the temporal gyrus underlying depression in Alzheimer's disease patients. Front. Aging Neurosci. 10:37. doi: 10.3389/fnagi.2018.00037

Lowe, M. J., Mock, B. J., and Sorenson, J. A. (1998). Functional connectivity in single and multislice echoplanar imaging using resting-state fluctuations. Neuroimage 7, 119-132. doi: 10.1006/nimg.1997.0315

Maass, A., Landau, S., Baker, S. L., Horng, A., Lockhart, S. N., Joie, R., et al. (2017). Comparison of multiple tau-PET measures as biomarkers in aging and Alzheimer's Disease. Neuroimage 157, 448-463. doi: 10.1016/j.neuroimage. 2017.05.058

Maruszak, A., and Thuret, S. (2014). Why looking at the whole hippocampus is not enough-a critical role for anteroposterior axis, subfield and activation analyses to enhance predictive value of hippocampal changes for Alzheimer's disease diagnosis. Front. Cell. Neurosci. 8:95. doi: 10.3389/fncel.2014.00095

Nakashiba, T., Young, J. Z., Mchugh, T. J., and Buhl, D. L. (2008). Tonegawa S. Transgenic inhibition of synaptic transmission reveals role of CA3 output in hippocampal learning. Science 319, 1260-1264. doi: 10.1126/science.1151120

Nakazawa, K., Quirk, M. C., Chitwood, R. A., Watanabe, M., Yeckel, M. F., Sun, L. D., et al. (2002). Requirement for hippocampal CA3 NMDA receptors in associative memory recall. Science 297, 211-218. doi: 10.1126/science.1071795

Okada, K., and Okaichi, H. (2009). Functional differentiation and cooperation among the hippocampal subregions in rats to effect spatial memory processes. Behav. Brain Res. 200, 181-191. doi: 10.1016/j.bbr.2009.01.011

Oliva, A., Fernandez-Ruiz, A., Buzsaki, G., and Berenyi, A. (2016). Role of hippocampal CA2 region in triggering sharp-wave ripples. Neuron 91, 1342 1355. doi: 10.1016/j.neuron.2016.08.008

Petersen, R. C. (2011). Clinical practice. Mild cognitive impairment. N. Engl. J. Med. 364, 2227-2234. doi: 10.1056/NEJMcp0910237

Petersen, R. C., Stevens, J. C., Ganguli, M., Tangalos, E. G., Cummings, J. L., and Dekosky, S. T. (2001). Practice parameter: early detection of dementia: mild cognitive impairment (an evidence-based review). Report of the quality standards subcommittee of the American Academy of Neurology. Neurology 56, 1133-1142. doi: 10.1212/WNL.56.9.1133

Raettig, T., and Kotz, S. A. (2008). Auditory processing of different types of pseudowords: an event-related fMRI study. Neuroimage 39, 1420-1428. doi: 10.1016/j. neuroimage.2007.09.030

Ranganath, C., Heller, A., Cohen, M. X., Brozinsky, C. J., and Rissman, J. (2005). Functional connectivity with the hippocampus during successful memory formation. Hippocampus 15, 997-1005. doi: 10.1002/hipo.20141

Rolls, E. T., and Kesner, R. P. (2006). A computational theory of hippocampal function, and empirical tests of the theory. Prog. Neurobiol. 79, 1-48. doi: 10.1016/j.pneurobio.2006.04.005
Saad, Z. S., Reynolds, R. C., Jo, H. J., Gotts, S. J., Chen, G., Martin, A., et al. (2013). Correcting brain-wide correlation differences in resting-state FMRI. Brain Connect. 3, 339-352. doi: 10.1089/brain.2013.0156

Scheltens, P., Leys, D., Barkhof, F., Huglo, D., Weinstein, H. C., Vermersch, P., et al. (1992). Atrophy of medial temporal lobes on MRI in "probable" Alzheimer's disease and normal ageing: diagnostic value and neuropsychological correlates. J. Neurol. Neurosurg. Psychiatry 55, 967-972. doi: 10.1136/jnnp.55.10.967

Schonheit, B., and Zarski, R. (2004). Ohm TG. Spatial and temporal relationships between plaques and tangles in Alzheimer-pathology. Neurobiol. Aging 25, 697-711. doi: 10.1016/j.neurobiolaging.2003.09.009

Shah, P., Bassett, D. S., Wisse, L., Detre, J. A., Stein, J. M., Yushkevich, P. A., et al. (2018). Mapping the structural and functional network architecture of the medial temporal lobe using 7T MRI. Hum. Brain Mapp. 39, 851-865. doi: $10.1002 / \mathrm{hbm} .23887$

Shipton, O. A., El-Gaby, M., Apergis-Schoute, J., Deisseroth, K., Bannerman, D. M., Paulsen, O., et al. (2014). Left-right dissociation of hippocampal memory processes in mice. Proc. Natl. Acad. Sci. U.S.A. 111, 15238-15243. doi: 10.1073/ pnas. 1405648111

Sormaz, M., Jefferies, E., Bernhardt, B. C., Karapanagiotidis, T., Mollo, G., Bernasconi, N., et al. (2017). Knowing what from where: hippocampal connectivity with temporoparietal cortex at rest is linked to individual differences in semantic and topographic memory. Neuroimage 152, 400-410. doi: 10.1016/j.neuroimage.2017.02.071

Tang, X., Holland, D., Dale, A. M., Younes, L., and Miller, M. I. (2015). The diffeomorphometry of regional shape change rates and its relevance to cognitive deterioration in mild cognitive impairment and Alzheimer's disease. Hum. Brain Mapp. 36, 2093-2117. doi: 10.1002/hbm.22758

Tsanov, M., and Manahan-Vaughan, D. (2008). Synaptic plasticity from visual cortex to hippocampus: systems integration in spatial information processing. Neuroscientist 14, 584-597. doi: 10.1177/1073858408315655

Tsien, J. Z., Huerta, P. T., and Tonegawa, S. (1996). The essential role of hippocampal CA1 NMDA receptor-dependent synaptic plasticity in spatial memory. Cell 87, 1327-1338. doi: 10.1016/S0092-8674(00) 81827-9

Ulrich, J. (1985). Alzheimer changes in nondemented patients younger than sixty-five: possible early stages of Alzheimer's disease and senile dementia of Alzheimer type. Ann. Neurol. 17, 273-277. doi: 10.1002/ana.410170309

Vandenberghe, R., Price, C., Wise, R., Josephs, O., and Frackowiak, R. S. (1996). Functional anatomy of a common semantic system for words and pictures. Nature 383, 254-256. doi: 10.1038/383254a0

Wallenstein, G. V., Eichenbaum, H., and Hasselmo, M. E. (1998). The hippocampus as an associator of discontiguous events. Trends Neurosci. 21, 317-323. doi: 10.1016/S0166-2236(97)01220-4

Whitfield-Gabrieli, S., and Nieto-Castanon, A. (2012). Conn: a functional connectivity toolbox for correlated and anticorrelated brain networks. Brain Connect. 2, 125-141. doi: 10.1089/brain.2012.0073

Yushkevich, P. A., Pluta, J. B., Wang, H., Xie, L., Ding, S. L., Gertje, E. C., et al. (2015). Automated volumetry and regional thickness analysis of hippocampal subfields and medial temporal cortical structures in mild cognitive impairment. Hum. Brain Mapp. 36, 258-287. doi: 10.1002/hbm. 22627

Conflict of Interest Statement: The authors declare that the research was conducted in the absence of any commercial or financial relationships that could be construed as a potential conflict of interest.

The reviewer HG and handling Editor declared their shared affiliation.

Copyright (C) $2018 \mathrm{Li}$, Jia, Qi, Fan, Ma, Pang, Ni, Li, Lu and Li. This is an open-access article distributed under the terms of the Creative Commons Attribution License (CC BY). The use, distribution or reproduction in other forums is permitted, provided the original author(s) and the copyright owner(s) are credited and that the original publication in this journal is cited, in accordance with accepted academic practice. No use, distribution or reproduction is permitted which does not comply with these terms. 